\title{
Short communication: Biogenic amine formation during fermentation in functional sheep milk yogurts
}

\author{
Vitor L. M. Silva, ${ }^{1}$ Marion P. Costa, ${ }^{2}$ Carla P. Vieira, ${ }^{3}$ and Carlos A. Conte-Junior ${ }^{1,3 *}$ \\ ${ }^{1}$ Universidade Federal Fluminense, Department of Food Technology, Faculdade de Veterinária, 24230-340, Niterói, Brazil \\ ${ }^{2}$ Universidade Federal da Bahia, Department of Preventive Veterinary Medicine and Animal Production, Escola de Medicina Veterinária, \\ 40170-110, Salvador, Brazil \\ ${ }^{3}$ Universidade Federal do Rio de Janeiro, Instituto de Química, Food Science Program, 21941-909, Rio de Janeiro, Brazil
}

\section{ABSTRACT}

The present study evaluated biogenic amine (BA) content during the fermentation period in functional sheep milk yogurts. Four treatments were prepared and assessed: natural (NSY), prebiotic (PreSY), probiotic (ProSY), and synbiotic (SynSY). Biogenic amines (putrescine, cadaverine, spermidine, spermine, and tyramine), proteolysis activity, and $\mathrm{pH}$ were measured during each hour of fermentation. Grumixama pulp was added to all formulations as a technological strategy and potential substrate for bacteria during fermentation. The yogurt and probiotic bacteria were viable $(\geq 7$ $\log \mathrm{cfu} \cdot \mathrm{mL}^{-1}$ ) on $\mathrm{d} 0$. The $\mathrm{pH}$ levels of the functional sheep milk yogurts had a more pronounced decrease than did the control of NSY. However, all yogurt samples underwent gradual decreases in $\mathrm{pH}$ until final fermentation. Proteolytic activity remained constant in all treatments during fermentation. The NSY, PreSY, ProSY, and SynSY presented the same behavior for all $\mathrm{BA}$, with differences in concentration. Putrescine, cadaverine, and spermidine contents decreased, whereas spermine remained constant and tyramine increased. We conclude that fermentation of functional sheep milk yogurts can produce tyramine.

Key words: fermented milk, Eugenia brasiliensis Lam., synbiotic, biogenic amine

\section{Short Communication}

Sheep milk contains higher contents of protein, lipids, minerals, and vitamins essential to human health than does cow milk. In addition, sheep milk has higher concentrations of CLA, which has beneficial effects on the human body (Park et al., 2007). Conjugated linoleic acid is considered a bioactive compound in milk that can confer the claim of functional food to sheep milk

Received January 26, 2019.

Accepted May 21, 2019.

*Corresponding author: carlosconte@id.uff.br derivatives such as yogurt. Functional foods have the potential to promote health through mechanisms not provided by conventional nutrition, and include bioactive compounds, probiotics, and prebiotics (Costa et al., 2013). The grumixama (Eugenia brasilienses Lam.) is a rustic plant growing throughout the southern and southeastern regions of Brazil. Its fruits are a source of bioactive compounds, including phenolic compounds, mainly flavonoids, and ellagitannins, which help biological systems to improve normal functions and health (Teixeira et al., 2018). In this experiment, grumixama pulp was added to all formulations of yogurt as a technological strategy and as a potential substrate for bacteria during fermentation. Probiotics are live microorganisms, and prebiotics are ingredients that are selectively fermented by specific microorganisms; both, when administered in adequate amounts, may benefit host health (Costa et al., 2013). However, some compounds present in foods, such as biogenic amines (BA), can be deleterious to human health due to vasoactive, psychoactive, and toxicological effects (Costa et al., 2018).

In foods of animal origin, BA are present in the raw material, because they have functions in vivo or because they are generated by microbial decarboxylation of amino acids. Thus, foods of animal origin are always susceptible to BA contamination. In addition, the types and concentrations of BA present are entirely related to the traits of the food and microbial decarboxylation. This is relevant mainly in foods such as yogurt, in which manufacturing demands a fermentation process. Thus in dairy products such as fermented milk, these compounds are currently detected, including tyramine, histamine, putrescine, cadaverine, and at lower concentrations, $\beta$-phenylethylamine and tryptamine (Costa et al., 2018). However, BA play relevant roles in human physiological functions. Thus, excessive consumption of $\mathrm{BA}$ is a concern even in functional products (Benkerroum, 2016) such as sheep milk yogurt. Several microorganisms, such as lactic acid bacteria, synthesize BA by amino acid decarboxylation. Therefore, at the end 
of fermentation and ripening, some lactic acid bacterial strains can produce these toxic compounds (Ladero et al., 2012). Moreover, there is currently a lack of data about BA behavior and concentration during sheep milk yogurt fermentation and storage, including the influence of probiotics and prebiotics. Thus, this study aimed to evaluate BA behavior during the fermentation process of functional sheep milk yogurts.

Raw sheep milk was obtained from healthy Lacaune sheep provided by Sítio Solidão (Miguel Pereira, Rio de Janeiro, Brazil; $\left.22^{\circ} 27^{\prime} 31.8^{\prime \prime} \mathrm{S}, 43^{\circ} 28^{\prime} 10.6^{\prime \prime} \mathrm{W}\right)$. Subsequently, at the laboratory, sheep milk was pasteurized at $90^{\circ} \mathrm{C}$ for $5 \mathrm{~min}$ and cooled to $43^{\circ} \mathrm{C}$ for addition of bacterial culture. Grumixama fruit pulp (Eugenia brasiliensis Lam.) was acquired from Sítio do Bello (Paraibuna, São Paulo, Brazil; $23^{\circ} 27^{\prime} 53.94^{\prime \prime} \mathrm{S}, 45^{\circ} 42^{\prime} 31.88^{\prime \prime} \mathrm{W}$ ), pasteurized and frozen. For yogurt preparation, the pulp was removed from the freezer and allowed to thaw at $4^{\circ} \mathrm{C}$ in a refrigerator.

The sheep milk yogurt was manufactured as described by Costa et al. (2015b). Pasteurized whole sheep milk was homogenized in a glass flask. Then, a thermophilic bacterial culture (1\% vol/vol; Lactobacillus delbrueckii ssp. bulgaricus and Streptococcus thermophilus; cat. YFL903, Chr. Hansen, Valinhos, Brazil) and 10\% (wt/vol) pasteurized grumixama pulp were added to all treatments. Four treatments were prepared: natural sheep yogurt (NSY) as control, a prebiotic added to the sheep yogurt (PreSY), a probiotic added (ProSY), and a synbiotic formulation containing both probiotic and prebiotic (SynSY). The prebiotic treatments (PreSY and SynSY) were supplemented with 5\% (wt/ vol) fructooligosaccharide (Fosvita, Sorocaba, São Paulo, Brazil). The probiotic treatments (ProSY and SynSY) were inoculated with $1 \times 10^{6} \mathrm{cfu} / \mathrm{g}$ Bifidobacterium animalis ssp. lactis (BB-12, Chr. Hansen). After homogenization, samples were incubated in an oven (HeraTherm, Thermo Fisher Scientific, Waltham, MA) at $43 \pm 11^{\circ} \mathrm{C}$. Two aliquots $(80 \mathrm{~mL})$ from each formulation were collected each hour during the fermentation period. The $\mathrm{pH}$ was measured and the samples were stored at $-20^{\circ} \mathrm{C}$ for future analysis. A pH value of 4.6 was considered to indicate the end of fermentation, and samples were refrigerated at $4 \pm 1^{\circ} \mathrm{C}$. The sheep milk yogurt manufacture was repeated 3 times, totaling 3 replicates $(\mathrm{n}=3)$.

Samples of S. thermophilus, L. delbrueckii ssp. bulgaricus, and B. animalis ssp. lactis (BB-12) were analyzed (d 0) to characterize the fermented product as yogurt and probiotic. The samples $(25 \mathrm{~g})$ were suspended in $225 \mathrm{~mL}$ of sterilized saline solution $0.1 \%$, homogenized, and subsequently spread on media selective for each strain. For spreading, the Eddy Jet 2 Spiral Plating System (IUL, S.A.; Barcelona, Spain) equipment was used. Streptococcus thermophilus were enumerated on M17 medium (Sigma-Aldrich, St. Louis, MO) with 1\% lactose after incubation at $37^{\circ} \mathrm{C}$ for $48 \mathrm{~h}$ under aerobiosis. Lactobacillus bulgaricus were enumerated on de Man, Rogosa, and Sharpe (MRS) agar (Himedia, Mumbai, India) with $\mathrm{pH}$ adjusted to 5.2 and incubation at $37^{\circ} \mathrm{C}$ for $72 \mathrm{~h}$ under anaerobiosis. Bifidobacterium lactis were enumerated on MRS agar supplemented with $10 \%$ neomycin sulfate stock solution, $3 \%$ nalidixic acid stock solution, $10 \% \mathrm{LiCl}$ stock solution, and $0.5 \% \mathrm{CyHCl}$ stock solution per liter of medium after incubation at $37^{\circ} \mathrm{C}$ for $72 \mathrm{~h}$ under anaerobiosis (Illupapalayam et al., 2014; Rezaei et al., 2014). Bacteriological analyses were performed in duplicate and expressed as log colonyforming units (CFU) per gram.

The $\mathrm{pH}$ was tested using a digital potentiometer (model PG1800; Cap-Lab Industry and Trade Ltd., São Paulo, Brazil). Proteolysis was measured as total free amino acids (TFAA). The TFAA content was determined according to the method described by Shori et al. (2013), with some modifications. Briefly, $10 \mathrm{~g}$ of each yogurt was homogenized with $40 \mathrm{~mL}$ of distilled water on a shaker at $40^{\circ} \mathrm{C}$ for $1 \mathrm{~h}$. The homogenate was then centrifuged at $5,500 \times g$ for $30 \mathrm{~min}$, and the supernatant was recovered and vortexed for $30 \mathrm{~s}$. The aqueous nitrogen extract $(200 \mu \mathrm{L})$ was homogenized with $2 \mathrm{~mL}$ of Zn-ninhydrin reagent $(0.8 \mathrm{~g}$ of ninhydrin was dissolved in a mixture of $80 \mathrm{~mL}$ of ethanol and $10 \mathrm{~mL}$ of acetic acid, both with a minimum of $99 \%$ purity), followed by addition of $1 \mathrm{~g}$ of zinc chloride dissolved in $1 \mathrm{~mL}$ of Milli-Q water (Millipore, Bedford, $\mathrm{MA}$ ). The homogenate was heated at $84^{\circ} \mathrm{C}$ for $5 \mathrm{~min}$ and cooled at room temperature. The TFAA content was estimated using the absorbance value at $490 \mathrm{~nm}$ against a tyrosine standard curve with a UV-1800 spectrophotometer (Shimadzu, Kyoto, Japan) and using a mixture of $200 \mu \mathrm{L}$ of Milli-Q water (Millipore) with $2 \mathrm{~mL}$ of Zn-ninhydrin reagent as blank. Results were expressed as concentrations $(\mathrm{m} M)$.

Extraction, derivatization, and chromatographic analysis of BA were undertaken according to the method proposed by Vieira et al. (2017). The chromatographic system consisted of an LC-20AT pump, SPD-M20A diode array detector, CTO-20A oven, and SIL-20AC autosampler, all of which were connected to a CBM-20A controller (Shimadzu, Kyoto, Japan). Each extracted and derivatized sample was injected 3 times $(\mathrm{n}=3)$. The BA were identified by retention time and quantified by peak area using standard curves in the matrix integrated into LC Solution software and expressed as milligrams per liter.

This experiment was repeated 3 times $(\mathrm{n}=3)$. Independent factors, refrigerated storage, and their combined effects were evaluated using ANOVA. When a 
Table 1. Bacterial count (log cfu per gram) in sheep milk yogurts prior to fermentation (d 0)

\begin{tabular}{lccc}
\hline Sample $^{1}$ & $\begin{array}{c}\text { Streptococcus } \\
\text { thermophilus }\end{array}$ & $\begin{array}{c}\text { Lactobacillus } \\
\text { bulgaricus }\end{array}$ & $\begin{array}{c}\text { Bifidobacterium } \\
\text { lactis }\end{array}$ \\
\hline NSY & $10.40 \pm 0.05^{\mathrm{a}}$ & $7.80 \pm 0.90^{\mathrm{a}}$ & $\mathrm{ND}^{2}$ \\
PreSY & $10.17 \pm 0.08^{\mathrm{a}}$ & $7.17 \pm 0.49^{\mathrm{a}}$ & $\mathrm{ND}^{\mathrm{a}}$ \\
ProSY & $10.47 \pm 0.11^{\mathrm{a}}$ & $7.67 \pm 0.45^{\mathrm{a}}$ & $7.00 \pm 0.66^{\mathrm{a}}$ \\
SynSY & $10.30 \pm 0.17^{\mathrm{a}}$ & $8.34 \pm 0.61^{\mathrm{a}}$ & $7.03 \pm 0.74^{\mathrm{a}}$
\end{tabular}

${ }^{a}$ Different lowercase superscripts in the same column indicate significant differences $(P<0.05)$.

${ }^{1} \mathrm{NSY}=$ natural sheep milk yogurt, control; PreSY = prebiotic-treated sheep milk yogurt; ProSY = probiotic-treated sheep milk yogurt SynSY = synbiotic-treated sheep milk yogurt. Results are expressed as mean \pm standard deviation $(\mathrm{n}=3)$.

${ }^{2} \mathrm{ND}=$ not detected.

difference was detected at $95 \%$ confidence interval $(P<$ 0.05 ), Tukey's test was performed using XLSTAT software (version 2016.02.28451; Addinsoft, Paris, France).

The results of bacterial counts in the different formulations of sheep milk yogurts are presented in Table 1. Bacterial counts of $S$. thermophilus and L. bulgaricus differed because of the synergistic dynamics of the commercial coculture (Costa et al., 2015b; Silva et al., 2017). Initial values of $B$. lactis indicated viability in the probiotic yogurts $(\geq 7 \log \mathrm{cfu} / \mathrm{g})$. Nevertheless, all 3 bacteria counts rose to levels that would characterize the fermented milk as yogurt by Codex Alimentarius standards (Codex Alimentarius, 2010) in all treatments (NSY, PreSY, ProSY, and SynSY) and as probiotic yogurts in the cases of ProSY and SynSY. Thus, we found that the addition of grumixama pulp did not influence the viability of the starter culture or probiotic.

The $\mathrm{pH}$ of raw sheep milk was around $6.32 \pm 0.05$, which decreased in all treatments (NSY, PreSY, ProSY, and SynSY) during the fermentation period (Figure 1). The decrease $(P<0.05)$ in milk $\mathrm{pH}$ during yogurt fermentation is related to lactose hydrolysis and production of organic acids (Costa and Conte-Junior, 2015). In addition, according to Costa et al. (2016), who studied the fermentation of goat milk yogurts, this behavior is in accordance with growth of the starter culture and the probiotic bacteria (Table 1). Bacterial metabolic activity ferments lactose and produces lactic acid, which decreases the $\mathrm{pH}$ of the yogurt (Gaspar et al., 2013). After $3 \mathrm{~h}$ of fermentation, the treatments with prebiotic, probiotic, or both (PreSY, ProSY, and SynSY) showed a more pronounced decrease in $\mathrm{pH}$ than did the control sheep milk yogurt (NSY). This difference in $\mathrm{pH}$ decline may be related to the higher activity of the cultures present and to the presence of fermented ingredients.

Concentrations of TFAA in each treatment are reported in Figure 2. The TFAA content remained constant in all treatments during fermentation, varying

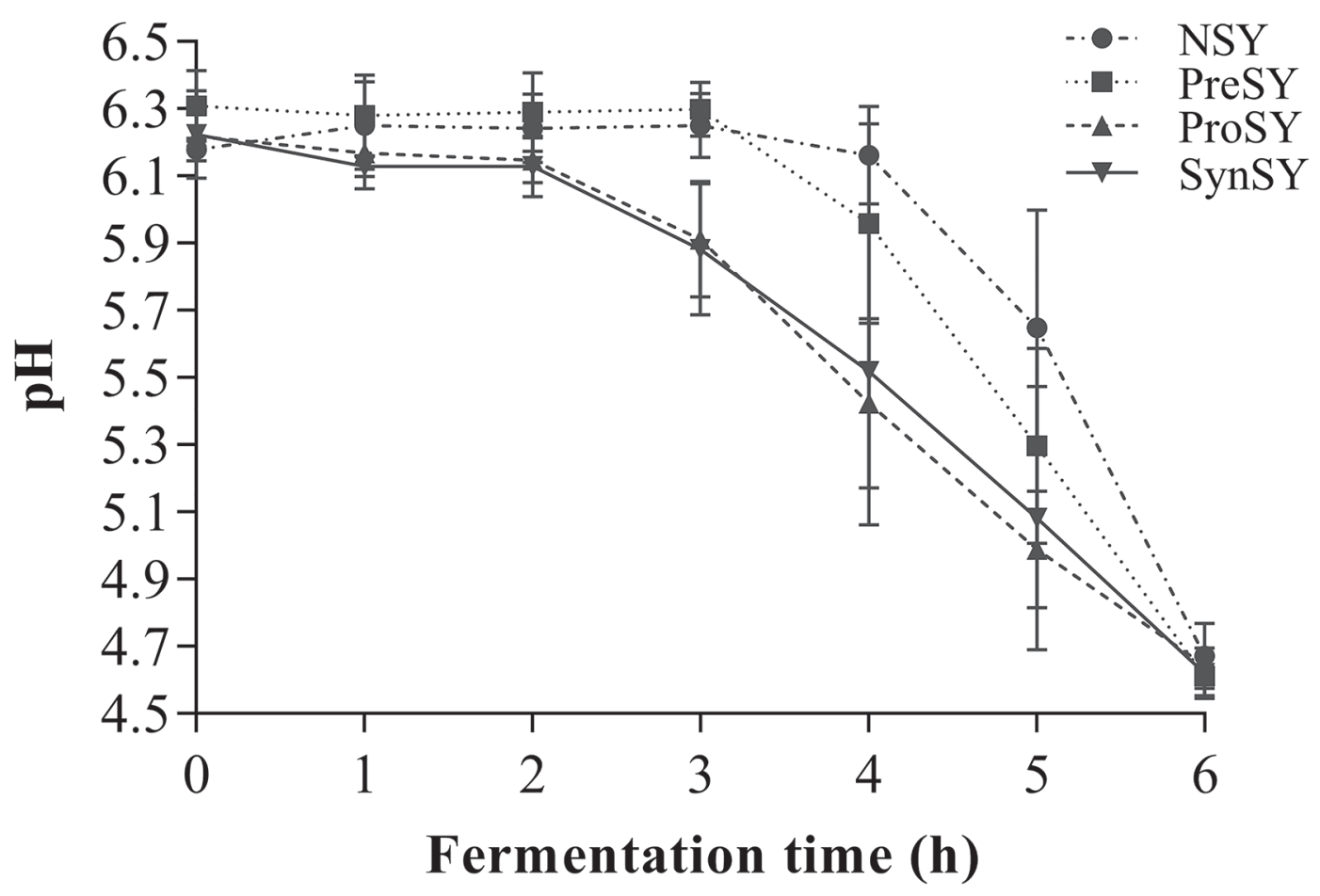

Figure 1. Changes in $\mathrm{pH}$ levels (mean $\pm \mathrm{SD}$ ) during fermentation of sheep milk yogurts. NSY $=$ natural sheep milk yogurt; PreSY $=$ prebiotic sheep milk yogurt; ProSY = probiotic sheep milk yogurt; SynSY = synbiotic sheep milk yogurt. 


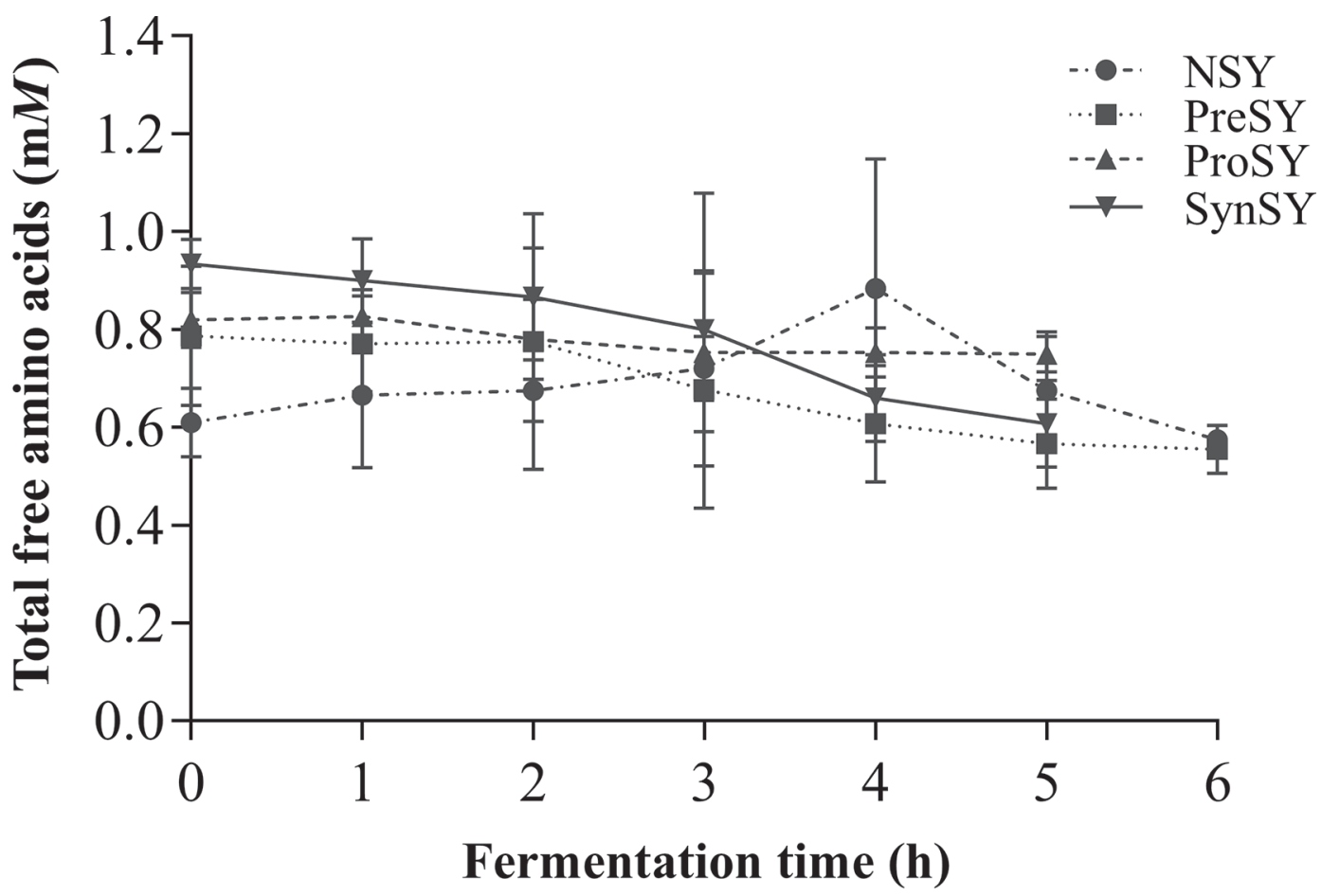

Figure 2. Proteolysis during fermentation of sheep milk yogurts, measured in levels of total free amino acids (TFAA; m $M$; mean \pm SD). NSY $=$ natural sheep milk yogurt; PreSY = prebiotic sheep milk yogurt; ProSY = probiotic sheep milk yogurt; SynSY = synbiotic sheep milk yogurt.

between 0.6 to $1.0 \mathrm{~m} M$. According to El-Zahar et al. (2003), an initial increase of TFAA in sheep milk yogurt occurs because of the developing proteolytic activity by the starter culture. However, TFAA levels generally drop after $4 \mathrm{~h}$ of fermentation, as TFAA are consumed by the growing microorganisms. In this study, the constant values of TFAA in sheep milk yogurt indicate that these nitrogenous compounds are both consumed and produced during the fermentation period by yogurt and probiotic bacteria growth. Moreover, we found no difference between treatments with regard to proteolysis (Figure 2).

Concentrations of BA in the tested sheep milk yogurts are shown in Table 2. Across the fermentation process, all BA (putrescine, cadaverine, spermidine, spermine, and tyramine) exhibited similar behaviors in all treatments (NSY, PreSY, ProSY, and SynSY). In general, concentrations of putrescine, cadaverine, and spermidine decreased, whereas that of spermine remained constant and tyramine increased $(P<0.05)$ during fermentation. The reduction in putrescine can be related to the ability of lactic acid bacteria to degrade BA through an enzymatic pathway regulated to oxidase enzymes (Costa et al., 2015a, 2018). Spermine and spermidine are synthesized endogenously, being present in raw milk. However, these amines can be produced and consumed in food during fermentation and storage
(Costa et al., 2018). The reduction in cadaverine may be attributed to the presence of Lactobacillus, which degrades cadaverine (Tosukhowong et al., 2011). In addition, S. thermophilus and Lactobacillus spp. are low cadaverine producers (Gezginc et al., 2013).

In contrast, tyramine is synthesized by $S$. thermophilus and L. acidophilus; both have been reported as significant tyramine producers in dairy products (Benkerroum, 2016). Across treatments, despite similar behaviors, there were differences $(P<0.05)$ in concentrations of BA during fermentation. During fermentation, some particularities (such as the behavior of putrescine in PreSY) can be correlated mainly with the ratios of AA in each treatment, the presence or absence of prebiotics and probiotics, and the rate of proteolysis velocity (Costa et al., 2015a).

Even at this first assessment, we observe that the fermentation of functional sheep milk yogurts contributes to the formation of tyramine. However, further studies must be carried out to determine the behavior of BA during storage, as well as to evaluate the long-term effects of the consumption of BA at these concentrations in functional foods. This line of research is important to elucidate to the academy and the dairy industry the risks of yogurt contamination with BA during manufacture, even in functional products. In addition, due to the potential toxicity of BA to human health, evalu- 


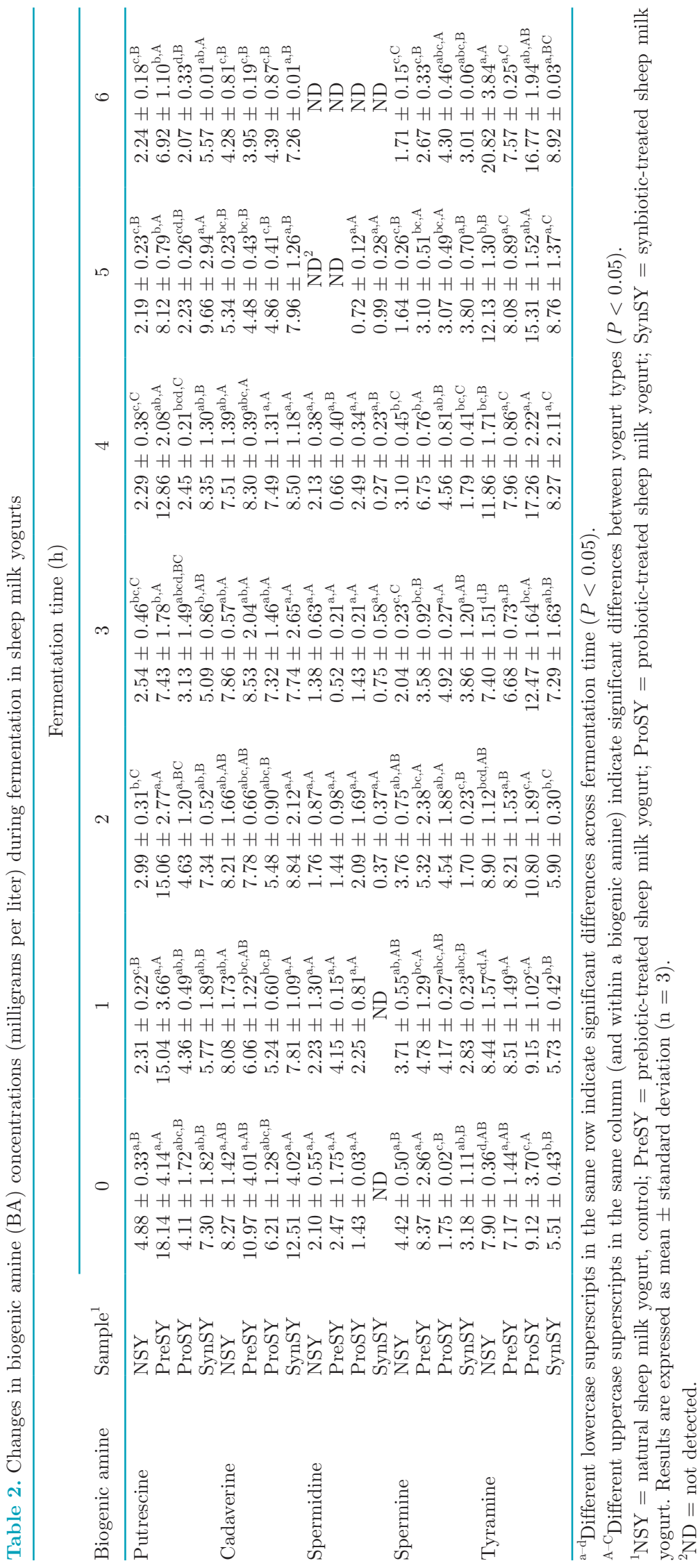


ation of BA in such types of dairy products is essential to clarify the behavior of BA during storage.

\section{ACKNOWLEDGMENTS}

The authors are grateful for the financial support provided by the Coordenação de Aperfeiçoamento de Pessoal de Nível Superior (CAPES, Brasília, Brazil), the Fundação de Amparo à Pesquisa do Estado do Rio de Janeiro (process no. E-26/201.185/2014, E-26/010.001.911/2015, and E-26/010.000274/2017, FAPERJ, Rio de Janeiro, Brazil), and the Conselho Nacional de Desenvolvimento Científico e Tecnológico (process no. 311361/2013-7, 400136/2014-7, and 166186/2015-5, CNPq, Brasília, Brazil). Milk samples were supplied by Sítio Solidão (Miguel Pereira, Rio de Janeiro, Brazil).

\section{REFERENCES}

Benkerroum, N. 2016. Biogenic amines in dairy products: Origin, incidence, and control means. Compr. Rev. Food Sci. Food Saf. $15: 801-826$.

Codex Alimentarius. 2010. Codex standard for fermented milks. Standards 243-2003 in Codex Alimentarius: Milk and Milk Products, 2nd ed. Codex Alimentarius Commission, Brussels, Belgium.

Costa, M. P., and C. A. Conte-Junior. 2015. Chromatographic methods for the determination of carbohydrates and organic acids in foods of animal origin. Compr. Rev. Food Sci. Food Saf. 14:586-600.

Costa, M. P., C. F. Balthazar, R. V. de B. Pinto, A. G. Cruz, and C. A. Conte-Junior. 2013. Leite fermentado: Potencial alimento funcional. Encicl. Biosf. 9:1387-1408.

Costa, M. P., C. F. Balthazar, B. L. Rodrigues, C. A. Lazaro, A. C. Silva, A. G. Cruz, and C. A. Conte-Junior. 2015a. Determination of biogenic amines by high-performance liquid chromatography (HPLC-DAD) in probiotic cow's and goat's fermented milks and acceptance. Food Sci. Nutr. 3:172-178.

Costa, M. P., B. da Silva Frasao, B. R. C. da Costa Lima, B. L. Rodrigues, and C. A. C. Conte-Junior. 2016. Simultaneous analysis of carbohydrates and organic acids by HPLC-DAD-RI for monitoring goat's milk yogurts fermentation. Talanta. 152:162-170.

Costa, M. P., B. S. Frasao, A. C. Silva, M. Q. Freitas, R. M. Franco, and C. A. Conte-Junior. 2015b. Cupuassu (Theobroma grandiflorum) pulp, probiotic, and prebiotic: Influence on color, apparent viscosity, and texture of goat milk yogurts. J. Dairy Sci. 98:59956003.
Costa, M. P., B. L. Rodrigues, B. S. Frasao, and C. A. Conte-Junior. 2018. Biogenic amines as food quality index and chemical risk for human consumption. Pages $75-108$ in Food Quality: Balancing Health and Disease. A. M. Grumezescu and A. M. Holban, ed. Academic Press, London, UK.

El-Zahar, K., J.-M. Chobert, M. Sitohy, M. Dalgalarrondo, and T. Haertlé. 2003. Proteolytic degradation of ewe milk proteins during fermentation of yoghurts and storage. Food/Nahrung. 47:199-206.

Gaspar, P., A. L. Carvalho, S. Vinga, H. Santos, and A. R. Neves. 2013. From physiology to systems metabolic engineering for the production of biochemicals by lactic acid bacteria. Biotechnol. Adv. 31:764-788.

Gezginc, Y., I. Akyol, E. Kuley, and F. Özogul. 2013. Biogenic amines formation in Streptococcus thermophilus isolated from home-made natural yogurt. Food Chem. 138:655-662.

Illupapalayam, V. V., S. C. Smith, and S. Gamlath. 2014. Consumer acceptability and antioxidant potential of probiotic-yogurt with spices. Lebensm. Wiss. Technol. 55:255-262.

Ladero, V., M. Fernández, M. Calles-Enríquez, E. Sánchez-Llana, E. Cañedo, M. C. Martín, and M. A. Alvarez. 2012. Is the production of the biogenic amines tyramine and putrescine a species-level trait in enterococci? Food Microbiol. 30:132-138.

Park, Y. W., M. Juárez, M. Ramos, and G. F. W. Haenlein. 2007. Physico-chemical characteristics of goat and sheep milk. Small Rumin. Res. 68:88-113.

Rezaei, R., M. Khomeiri, M. Aalami, and M. Kashaninejad. 2014. Effect of inulin on the physicochemical properties, flow behavior and probiotic survival of frozen yogurt. J. Food Sci. Technol. 51:2809-2814.

Shori, A. B., A. S. Baba, and P. F. Chuah. 2013. The effects of fish collagen on the proteolysis of milk proteins, ACE inhibitory activity and sensory evaluation of plain- and Allium sativum-yogurt. J. Taiwan Inst. Chem. Eng. 44:701-706.

Silva, F. A., M. E. G. de Oliveira, R. M. F. de Figueirêdo, K. B. Sampaio, E. L. de Souza, C. E. V. de Oliveira, M. M. E. Pintado, and R. C. R. E. Queiroga. 2017. The effect of Isabel grape addition on the physicochemical, microbiological and sensory characteristics of probiotic goat milk yogurt. Food Funct. 8:2121-2132.

Teixeira, L. de L., N. M. A. Hassimotto, and F. M. Lajolo. 2018. Grumixama-Eugenia brasiliensis Lam. Pages 219-224 in Exotic Fruits. S. Rodrigues, E. de Oliveira Silva, and E. S. de Brito, ed. Academic Press, London, UK.

Tosukhowong, A., W. Visessanguan, L. Pumpuang, P. Tepkasikul, A. Panya, and R. Valyasevi. 2011. Biogenic amine formation in Nham, a Thai fermented sausage, and the reduction by commercial starter culture, Lactobacillus plantarum BCC 9546. Food Chem. 129:846-853.

Vieira, C. P., M. P. da Costa, V. L. Silva, B. da Silva Frasao, L. F. C. de Aquino, Y. E. de Oliveira Nunes, and C. A. Conte-Junior. 2017. Development and validation of RP-HPLC-DAD method for biogenic amines determination in probiotic yogurts. Arab. J. Chem. In press. 